\title{
DISTRIBUIÇÃO ESPACIAL DO ENTEROPARASITISMO EM USUÁRIOS DO SISTEMA ÚNICO DE SAÚDE, EM LIMOEIRO DO NORTE, CEARÁ, BRASIL, ENTRE 2009 E 2014
}

\author{
SPATIAL DISTRIBUTION OF INTESTINAL PARASITISM IN \\ USERS OF SISTEMA ÚNICO DE SAÚDE, IN LIMOEIRO DO \\ NORTE, CEARÁ, BRAZIL, BETWEEN 2009 AND 2014 \\ DISTRIBUCIÓN ESPACIAL DE PARASITISMO INTESTINAL EN \\ LOS USUARIOS DEL SISTEMA ÚNICO DE SALUD, EN LIMOEIRO \\ DO NORTE, CEARÁ, BRASIL, ENTRE 2009 Y 2014 \\ Carlos Vangerre de Almeida Maia - Instituto Centro de Ensino Tecnológico - Fortaleza - Ceará - Brasil \\ cvamaia@yahoo.com.br \\ Gustavo Souza Valladares - Universidade Federal do Piauí - Teresina - Piauí - Brasil \\ valladares@ufpi.edu.br \\ Izabella Cabral Hassum - Empresa Brasileira de Pesquisa Agropecuária - Teresina - Piauí - Brasil
izabella.cabral@hotmail.com
}

Resumo

0 enteroparasitismo é um agravo de amplitude global, possuindo relação com as condições ambientais, guardando diferenças inter- e intrarregionais. Os Sistemas de Informações Geográficas (SIG) podem auxiliar 0 planejamento que almeje seu controle. 0 objetivo desta pesquisa foi espacializar a prevalência enteroparasitária em usuários do Sistema Único de Saúde (SUS) em Limoeiro do Norte, Ceará, Brasil. Foram utilizados dados secundários, oriundos do Hospital Regional Dr. Deoclécio Lima Verde (HRDDLV), referente aos anos 2009 a 2014, realizando análise descritiva, mediante técnicas estatísticas com intervalo de confiança de $95 \%$. A prevalência vem sendo reduzida ao longo dos anos $(p<0,01)$; as mulheres foram as mais acometidas $(p<0,05)$, constatou-se maior prevalência das infecções protozoárias $(p<0,01) ; 0$ evento foi mais frequente entre aqueles que habitam a zona rural $(p<0,01)$, em especial, os pacientes residentes no território adstrito da Unidade Básica de Saúde (UBS) denominada Serra $(p<0,01) .0$ uso do SIG, mediante produção de mapa temático, mostrou ser de grande valia para a identificação de áreas de risco, sendo, uma ferramenta que pode auxiliar a gestão no planejamento visando o controle dessa infecção.

Palavras-chave: parasitoses intestinais, geoprocessamento, áreas de risco.

\section{Abstract}

The enteroparasitism is a global scale problem having a close relation with the environmental conditions, and keeping inter- and intra-regional differences. The Geographic Information System (GIS) can help the planning that aims to control intestinal parasites. The objective of this research was to locate the prevalence of endoparasitosis in users of the Health Unified System (Sistema Único de Saúde - SUS) in Limoeiro do Norte, Ceará, Brazil. Secondary data from the Regional Hospital Dr. Deoclécio Lima Verde from the years 2009-2014 were used, executing descriptive analysis by statistical techniques with a confidence interval of $95 \%$. The prevalence has been reduced over the years $(p<0.01)$; women are the most affected $(p<0.05)$; there was 
a higher protozoa infection prevalence $(p<0.01)$; the event is more frequent among those who inhabit the rural area $(p<0.01)$, in particular, patients living in the territory around Health Basic Unit (Unidade Básica de Saúde - UBS) called Serra $(p<0.01)$. The use of GIS, through the production of a thematic map, proved to be of great value to the identification of risk areas, being therefore a tool that can assist management in conducting planning that seeks to control this infection.

Keywords: intestinal parasites, geoprocessing, risk areas.

\section{Resumen}

El parasitismo intestinal es un agravio mundial, que tiene relación con las condiciones ambientales, guardando diferencias inter e intrarregionales. Los Sistemas de Información Geográfica (SIG) pueden ayudar en la planificación que busque su control. El objetivo de esta investigación fue espacializar la prevalencia de parásitos intestinales en usuarios del Sistema Único de Salud (SUS) en Limoeiro do Norte, Ceará, Brasil. Fueron utilizados datos secundarios del Hospital Regional Dr. Deoclécio Lima Verde (HRDDLV), de los años 2009 a 2014, fueron realizados análisis descriptivos, mediante procedimientos estadísticos con un intervalo de confianza de $95 \%$. La prevalencia se ha reducido a lo largo de los años $(p<0,01)$; las mujeres fueron las más afectados $(p<0,05)$, se constató mayor prevalencia de infecciones por protozoos $(p<0,01)$; el evento fue más común entre los que viven en el campo $(p<0,01)$, en particular, los pacientes que viven en el territorio adjunto de la Unidad Básica de Salud (UBS) denominada Serra $(p<0,01)$. El uso del SIG, mediante la producción de mapas temáticos, demostró ser de gran valor para la identificación de zonas de riesgo, siendo una herramienta que puede ayudar a la administración en la planificación, para el control de esa infección. Palabras-clave: parasitosis intestinales, geoprocesamiento, zonas de riesgo.

\section{Introdução}

O parasitismo intestinal é o estado de infecção cuja agressão repercute prejudicialmente sobre o hospedeiro, causado, mormente, por protozoários e helmintos. Mais recorrente na África, Ásia e América Latina, ocorrendo em áreas urbanas (principalmente em periferias e favelas) e rurais, presente em todas as faixas etárias, constituindo indicador de status socioeconômico, de adequabilidade de serviços de saneamento, incolumidade e de saúde pública de uma região (Bellin; Grazziotin, 2011; Belo et al., 2012; Fernandes et al., 2011; Lodo et al., 2010; Santos; Merlini, 2010; Silva et al., 2010; Silva et al., 2009).

O endoparasitismo não é uma relação binomial, depende, pelo menos, de três fatores: o parasito, o hospedeiro e o meio onde ocorre a relação. Considerando que o ambiente possui contígua ligação com o agravo, é interessante que o espaço seja analisado, recaindo sobre si observação estratégica, onde a utilização do Sistema de Informação Geográfica (SIG) pode contribuir para o entendimento integrado de informações de caráter epidemiológico, ambiental e socioeconômico, auxiliando, para Hino et al. (2011), na identificação de áreas geográficas e populações que estão mais susceptíveis a algum risco. 
Portanto, o objetivo dessa pesquisa é especializar a prevalência de enteroparasitoses nos territórios adstritos das Unidades Básicas de Saúde (UBSs) de Limoeiro do Norte, Ceará, Brasil, entre 2009 e 2014, sob a hipótese de que o enteroparasitismo ocorre de maneira heterogênea no município, havendo, pois, áreas de risco e populações mais vulneráveis.

Materiais e métodos

O estudo é do tipo ecológico e descritivo, com abordagem quantitativa, realizado em Limoeiro do Norte (Figura 1), situado a $200 \mathrm{~km}$ a sudeste de Fortaleza, capital do estado, com a $25^{\mathrm{a}}$ maior população do Ceará - 56.281. Desses, 32.502 (57,75\%) residem no perímetro urbano, habitando 11.058 domicílios (57,43\%) (IBGE, 2010).
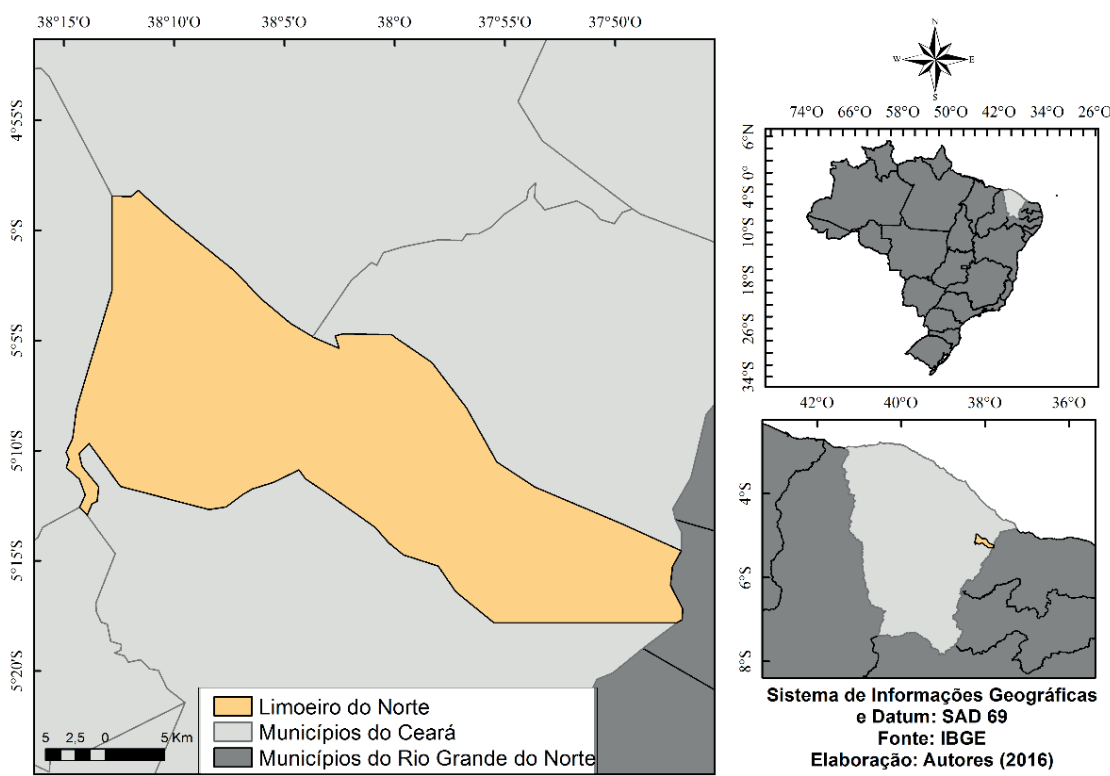

Figura 1 - Localização do município de Limoeiro do Norte, Ceará, Brasil

Conforme Perfil Básico Municipal de 2013, o clima de Limoeiro do Norte é Tropical Quente Semiárido, com pluviosidade anual de 720,5 
$\mathrm{mm}$, temperatura média entre $26^{\circ}$ e $28^{\circ}$ e período chuvoso de janeiro a abril (Ceará, 2013).

Em 2014, o município estava regionalizado em 15 territórios adstritos às UBSs (Figura 2), sendo sete na zona urbana (Centro, Antônio Holanda, Populares, Centro II, Luís Alves, Boa Fé e Bom Nome) e oito na zona rural (Várzea do Cobra, Arraial, Bixopá, Córrego de Areia, Setores, Cabeça Preta, Serra e Espinho).
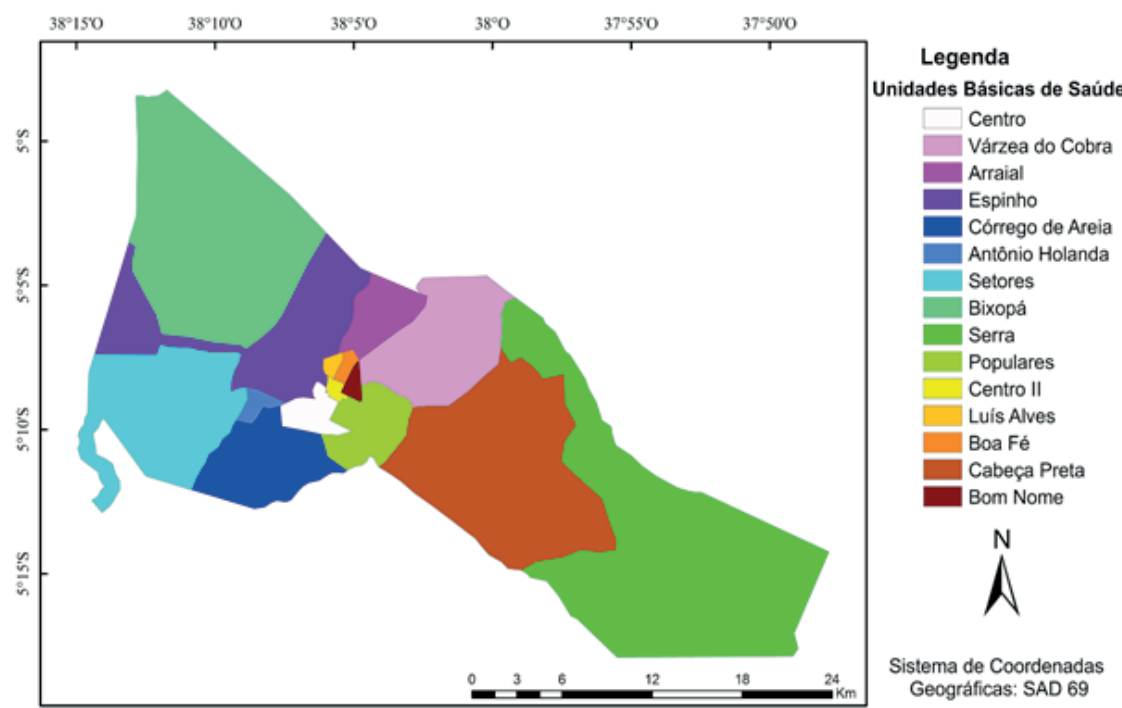

Figura 2 - Territórios adstritos às Unidades Básicas de Saúde em Limoeiro do Norte, Ceará, Brasil

Fonte: Autores (2016)

No município, o Hospital Regional Dr. Deoclécio Lima Verde (HRDDLV) dispõe de um software gerencial denominado Info Hospi (Limoeiro do Norte, 2009), que possibilita a confecção de relatórios referente aos procedimentos realizados.

Para o presente estudo, utilizaram-se os dados secundários oriundos dos relatórios dos exames coproscópicos realizados no HRDDLV, mediante informações do Info Hospi, para os anos de 2009 a 2014, de onde foram colhidas informações sobre: idade, sexo, UBS de origem, além do resultado 
e identificação parasitária dos pacientes que buscaram o Sistema Único de Saúde (SUS) para realizar o procedimento laboratorial.

Após a identificação dos parasitos, estes foram dicotomizados em: protozoários e helmintos. Para fins de análise, os parasitos Entamoeba hystolitica e Entamoeba dispar, foram considerados como uno, recebendo a nomenclatura $E$. hystolitica/dispar, pelo fato de que a técnica utilizada pelo laboratório - sedimentação espontânea (Hoffman; Pons; Janner, 1934), não permite distinção entre ambas.

Foram utilizadas as faixas etárias propostas pela Organização Mundial da Saúde (OMS) (apud Rocha; Braz; Calheiros, 2010): indivíduos com idade entre 0 e 9 anos (crianças); entre 10 e 19 (adolescentes); de 20 a 59 (adultos) e com idade acima de 60 anos (idosos), em países em desenvolvimento.

Portanto, os procedimentos que não contemplassem as informações correspondentes a: idade, sexo, território de origem do paciente, tipologia enteroparasitária e resultado do exame não foram considerados válidos para o presente estudo.

Para comparação de diferenças na distribuição enteroparasitária entre grupos, foi empregado o teste de Qui-quadrado $\left(\mathrm{X}^{2}\right)$ e para a identificação de áreas de risco, utilizou-se o Odds Ratio. O valor considerado para significância estatística foi de $5 \%(p<0,05)$, mesma significância adotada para identificar a variação da prevalência entre os anos, mediante Regressão Linear Simples.

A prevalência acumulada foi espacializada pelo programa ArcGis 10, utilizando-se a base cartográfica do município de Limoeiro do Norte, adaptada da base cartográfica do Ceará de 2009, fornecida pelo Instituto de Pesquisa e Estratégia Econômica do Ceará.

\section{Resultados e discussões}

Durante o período investigado, 3.824 procedimentos foram considerados válidos (95,24\%), dos quais 753 foram positivos para a ocorrência de endoparasito e/ou comensal, resultando em uma prevalência acumulada de $19,69 \%$.

A prevalência identificada foi inferior ao indicado pela OMS (25\%) e ao encontrado em Chapadinha - MA $(33,1 \%)$, porém superior ao diagnosticado em Sananduva - RS (8,1\%), ambos investigando usuários SUS (Silva et al., 2010; Bellin; Grazziotin, 2011). 
De acordo com Teixeira, Gomes e Souza (2012), a prevalência de doenças infecciosas é um forte indicativo de que algum ou todos os serviços de saneamento básico está fragilizado, coadunando com Heller (1997) sobre o entendimento de que há melhorias nos indicadores de saúde, mediante ampliação de cobertura de serviços de saneamento básico.

Entre 2009 e 2014, constatou-se redução média anual significativa (p $<0,001$ ) de $2,44 \%$ ao ano da prevalência (Figura 3), cuja redução pode estar relacionada com as melhorias sanitárias ocorridas no município, conforme observado por Maia, Hassum e Valladares (2014), que encontraram correlação inversamente proporcional entre a prevalência enteroparasitária e maior acesso aos serviços de saneamento básico entre os anos 2010 e 2013.

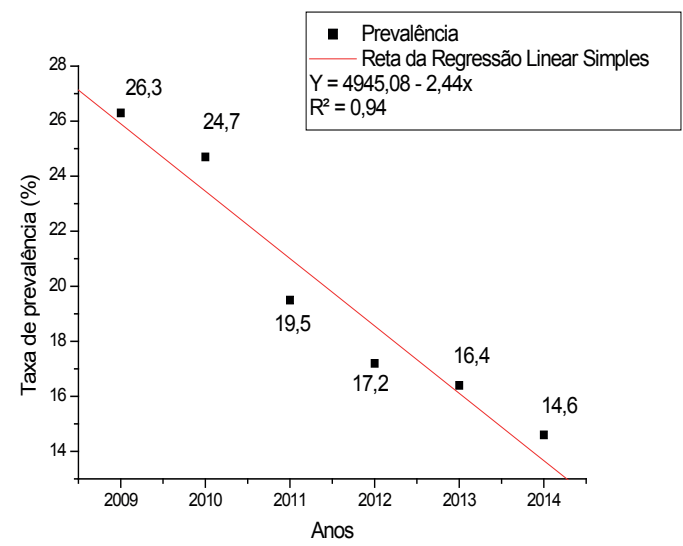

Figura 3 - Prevalência enteroparasitária (2009 - 2014), em Limoeiro do Norte, Ceará, Brasil Fonte: Elaboração própria.

Basso et al. (2008) encontraram redução de 1,4\% ao ano, durante 35 anos na prevalência endoparasitária em Caxias do Sul, RS, sendo as melhorias sanitárias justificativa para a redução da prevalência enteroparasitária de 89\%, em 1969, para 37\%, em 2004.

Dos pacientes que recorreram ao HRDDLV, as mulheres foram as mais frequentes - 2089 (54,64\%), assim como foi o sexo que apresentou maior acometimento - 401 (53,25\%), mas sem significância estatística ( $p=0,39)$.

Diferença de positividade entre os sexos pode ser justificada pelos hábitos e labor, inerentes a cada gênero. Habitualmente, as mulheres, por 
questões culturais, são as que mais procuram ajuda médica e são mais parasitadas (Gomes; Nascimento; Araújo., 2007).

Quando categorizados por faixa etária, constatou-se que os adultos foram os que mais buscaram o HRDDLV, assim como também foram os mais asseverados, porém sem significância estatística $(p=0,09)$ pelo teste de $\mathrm{X}^{2}$, conforme disposto na Tabela 1.

Tabela 1 - Enteroparasitismo em usuários do SUS, no município de Limoeiro do

Norte, Ceará, Brasil, de acordo com as faixas etárias, entre 2009 e 2014

\begin{tabular}{|c|c|c|c|}
\hline \multirow{2}{*}{ Faixa Etária } & \multicolumn{2}{|c|}{ Resultado } & \multirow{2}{*}{ Total (\%) } \\
\cline { 2 - 3 } & Negativo (\%) & Positivo (\%) & $1234(100 \%)$ \\
\hline Crianças & $990(80,23)$ & $244(19,77 \%)$ & $598(100 \%)$ \\
\hline Adolescentes & $476(79,6 \%)$ & $122(20,4 \%)$ & $1532(100 \%)$ \\
\hline Adultos & $1216(79,37 \%)$ & $316(20,63 \%)$ & $460(100 \%)$ \\
\hline Idosos & $389(84,57 \%)$ & $71(15,43 \%)$ & $3824(100 \%)$ \\
\hline Total & $3071(80,31 \%)$ & $753(19,69 \%)$ & \\
\hline
\end{tabular}

Fonte: Hospital Regional Dr. Deoclécio Lima Verde (adaptado).

Comparando os dados acima com outros trabalhos, observou-se discordância com o apresentado na literatura, onde é latente o maior acometimento em crianças (Castro; Peixoto; Rio., 2005; Mascarini et al., 2009).

Ao todo, foram identificados 928 parasitos e/ou comensais, cujos mais frequentes foram Endolimax nana (42,13\%), Entamoeba coli (25,53\%) e Giardia lamblia (21,22\%), prevalecendo infecção protozoótica (98,49\%).

Estes parasitos também foram os mais recorrentes nas pesquisas de Bellin e Grazziotin (2011), Matos e Cruz (2012), Lodo et al. (2010), Santos, Santos e Soares (2007) e por, Menezes et al. (2013), ambos envolvendo usuários do SUS.

A G.lamblia, dentre os mais prevalentes, é o que possui maior periculosidade, apresentando taxas variantes de prevalência nas diversas regiões brasileiras, fato que pode ser constatado em outras pesquisas coprológicas com usuários do SUS, como em Cumari, Goiás (GO), onde a prevalência deste parasito foi de 33,6\%, enquanto que em Goiorê, Paraná (PR), foi de 13,5\%. Em Chapadinha (MA) (25,4\%), em Macapá (AP), a frequência foi de 19,1\% e 14,9\% em Bom Jesus dos Perdões (SP) (Borges; Marciano; Oliveira, 2011; Oliveira; Chiuchetta, 2009; Silva Junior, 2010; Menezes et al., 2013, Lodo et al., 2010). 
Fregonesi et al. (2012) afirmam que este protozoário é apontado como a causa-mor das gastroenterites do mundo, onde cerca de $60 \%$ dos infectados, quando possuem sintomas, são inespecíficos, o que dificulta sua identificação.

Franco, Branco e Leal (2012) afirmam que nos últimos 25 anos, ocorreram cerca de 534 surtos epidêmicos de doenças de veiculação hídrica no planeta, sendo a G. lamblia o principal protozoário dentre os agentes etiológicos nestes eventos.

Em Limoeiro do Norte, os protozoários foram os mais frequentes, havendo diferença estatisticamente significativa pelo $\mathrm{X}^{2}(p<0,001)$; similar ao encontrado por Lodo et al., (2010) e por Matos e Cruz (2012) em pacientes atendidos em Bom Jesus dos Perdões (SP) e Ibiassucê (BA), onde foram encontradas prevalências protozoóticas acima de 93\%.

A baixa prevalência helmíntica pode estar associada ao uso indiscriminado de anti-helmínticos, encobrindo as reais condições sanitárias, ambientais e socioeconômicas da população, pois promove redução de prevalência, sem romper com seu ciclo epidemiológico, logo desverminando, mas deixando os indivíduos passíveis a reinfecções cíclicas, subestimando a prevalência (Belo et al., 2012; Frei; Juncansen; Ribeiro-Paes., 2008).

A prevalência subestimada também pode decorrer da técnica utilizada - sedimentação espontânea - a mais difundida na rede pública de saúde, capaz de detectar vários parasitos, mas por utilizar somente uma lâmina com amostra fecal, pode induzir a falsos-negativos, pois desconsidera a eliminação fecal intermitente (Mati; Pinto; Melo, 2011; Oro et al., 2010: Zaiden et al., 2008; Cunha et al., 2013).

Logo, concordando com Pezzi e Tavares (2007), é interessante a aplicação de mais de um método de análise para garantir maior fidedignidade do cenário, tendo em vista a variabilidade morfológica e biológica inerente ao parasito.

Alves et al. (2003), ao realizar inquérito coproscópico no semiárido piauiense, constataram baixa disseminação helmíntica nessa região, que apresenta baixo teor de umidade do solo, o que limita a proliferação de helmintos, logo evidenciando-se que características físico-químicas do solo podem repercutir sobre as helmintíases.

Considerando o enteroparasitismo como produto da tríade indivíduo, parasito e ambiente, resolveu-se categorizar a origem dos usuários do SUS quanto à região onde estes habitavam; se urbana, ou rural, conforme Tabela 2 . 
Tabela 2 - Prevalência enteroparasitária em usuários do SUS por região do município de Limoeiro do Norte, Ceará, Brasil, entre 2009 e 2014

\begin{tabular}{|c|c|c|c|}
\hline \multirow{2}{*}{ Região } & \multicolumn{2}{|c|}{ Resultado } & \multirow{2}{*}{ Total } \\
\cline { 2 - 3 } & Negativo (\%) & Positivo (\%) & $1997(52,22 \%)$ \\
\hline Rural & $1542(50,21 \%)$ & $455(60,42 \%)$ & $1827(47,78 \%)$ \\
\hline Urbano & $1529(49,79 \%)$ & $298(39,58 \%)$ & $3824(100 \%)$ \\
\hline Total & $3071(100 \%)$ & $753(100 \%)$ & \\
\hline
\end{tabular}

Fonte: Hospital Regional Dr. Deoclécio Lima Verde (Adaptado).

A hipótese da distribuição heterogênea do evento foi confirmada, cuja diferença entre as prevalências se mostrou significativa $(\mathrm{p}<0,001)$. Adenda-se que a medida de Odds Ratio foi igual a 0,661, ou seja: no período, os indivíduos que residiam na Zona Rural do município, possuíam, pelo menos, $66,1 \%$ a mais de chances de serem acometidos.

Diferenças entre a contaminação urbana e rural refletem as diferenças socioeconômicas, sanitárias e ambientais, uma vez que a cobertura de saneamento básico rural no Brasil é severamente incipiente ${ }^{1} \mathrm{e}$ foi aventado como um dos possíveis fatores para a maior prevalência rural em Itambé do Mato Dentro (MG) (Mati; Pinto; Melo,2011).

No município, de acordo com Maia, Hassum e Valladares (2014), 81,3\% da população urbana é atendida por água da rede geral, enquanto que 56,47\% da população rural usufrui desse serviço. Desigualdade também pode ser vista ao comparar coberturas de serviço de esgoto e coleta de resíduos, onde, na zona urbana, os índices atingem $86,1 \%$ e $11,16 \%$, enquanto que na zona rural, estes valores não ultrapassam 45,75\% e 1,1\%, respectivamente.

A literatura aponta que inadequações sanitárias incidem positivamente sobre a manifestação do endoparasitismo nas mais diversas partes do mundo (Pascual et al., 2010; Bermúdez et al., 2013; Abossie; Seid, 2014; Ngui et al., 2011).

Para Alves (2007), a exposição a bens e amenidades ambientais diferenciadas entre grupos populacionais, como qualidade da moradia e condições de saneamento básico, acarreta exposição a riscos, se configura como um caso de desigualdade ambiental.

De acordo com o Plano Municipal de Combate e Erradicação da Extrema Pobreza (Limoeiro do Norte, 2010), a zona rural do município, abrigava 3.463 (53,9\%) das 6.495 famílias em situação de extrema pobreza, logo é a região onde se encontra maior parte dos indivíduos 
economicamente mais hipossuficientes, que habitam as regiões mais insalubres, os tornando mais susceptíveis a eventos deletérios, evidenciando caso de injustiça ambiental, que pode ser entendido como grupos que arcam com maior parte dos danos e consequências oriundos das condições ambientais aos quais estão submetidos, refletindo nas condições de saúde e qualidade de vida (RBJA, 2002).

Após constatar distribuição heterogênea do agravo, buscou-se identificar quais os territórios que mais apresentaram risco para o processo infectocontagioso (Tabela 3).

Tabela 3 - Distribuição do enteroparasitismo em Limoeiro do Norte, Ceará, Brasil, de acordo com a UBS de procedência, entre 2009 e 2014

\begin{tabular}{|c|c|c|c|}
\hline \multirow{2}{*}{ UBS (Região) } & \multicolumn{2}{|c|}{ Resultado } & \multirow{2}{*}{ Total (\%) } \\
\cline { 2 - 3 } & Negativo (\%) & Positivo (\%) & \multirow{2}{*}{$263(100 \%)$} \\
\hline Centro (Urbano) & $221(84,03 \%)$ & $42(15,97 \%)$ & $343(100 \%)$ \\
\hline Várzea do Cobra (Rural) & $268(78,13 \%)$ & $75(21,87 \%)$ & $83(100 \%)$ \\
\hline Arraial (Rural) & $72(86,75 \%)$ & $11(13,25 \%)$ & $310(100 \%)$ \\
\hline Espinho (Rural) & $259(83,55 \%)$ & $51(16,45 \%)$ & $231(100 \%)$ \\
\hline Córrego de Areia (Rural) & $185(80,09 \%)$ & $46(19,91 \%)$ & $391(100 \%)$ \\
\hline Antônio Holanda (Urbano) & $338(86,45 \%)$ & $53(13,55 \%)$ & $347(100 \%)$ \\
\hline Setores (Rural) & $283(81,56 \%)$ & $64(18,44 \%)$ & $288(100 \%)$ \\
\hline Bixopá (Rural) & $218(75,69 \%)$ & $70(24,31 \%)$ & $332(100 \%)$ \\
\hline Serra (Rural) & $211(63,55 \%)$ & $121(36,45 \%)$ & $144(100 \%)$ \\
\hline Populares (Urbano) & $132(91,67 \%)$ & $12(8,33 \%)$ & $140(100 \%)$ \\
\hline Centro II (Urbano) & $122(87,14 \%)$ & $18(12,86 \%)$ & $247(100 \%)$ \\
\hline Luís Alves (Urbano) & $200(80,97 \%)$ & $47(19,03 \%)$ & $578(100 \%)$ \\
\hline Boa Fé (Urbano) & $464(80,28 \%)$ & $114(19,72 \%)$ & $63(100 \%)$ \\
\hline Cabeça Preta (Rural) & $46(73,02 \%)$ & $17(26,98 \%)$ & $64(100 \%)$ \\
\hline Bom Nome (Urbano) & $52(81,25 \%)$ & $12(18,75 \%)$ & $3824(100 \%)$ \\
\hline Total & $3071(80,31 \%)$ & $753(19,69 \%)$ & \\
\hline
\end{tabular}

Fonte: Hospital Regional Dr. Deoclécio Lima Verde (Adaptado).

Constatou-se que as maiores prevalências acumuladas foram encontradas em UBSs rurais, sendo elas Serra, Cabeça Preta, Bixopá e Várzea do Cobra. Houve diferença estatisticamente significativa $(p<0,001)$ entre as prevalências nos territórios, cuja distribuição espacial do agravo pode ser visualizada na Figura 4. 


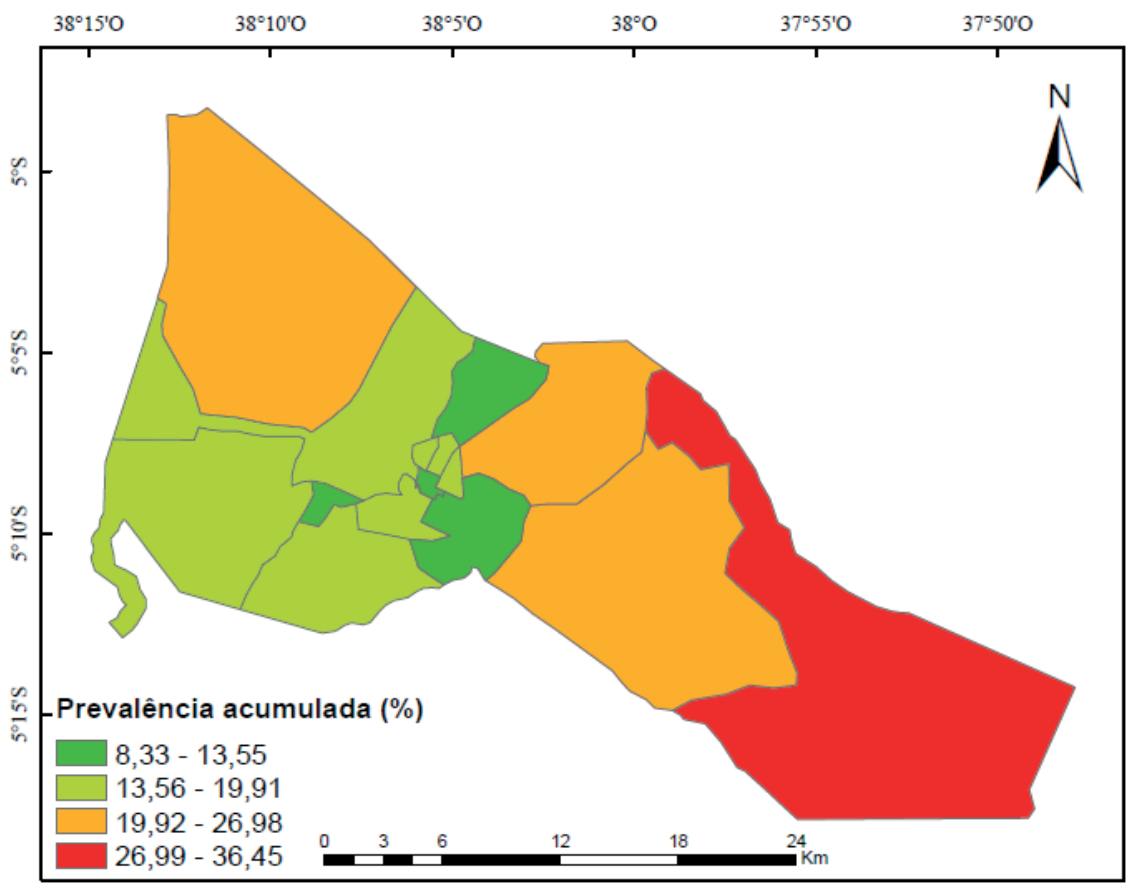

Figura 4 - Distribuição espacial da prevalência enteroparasitária acumulada no município de Limoeiro do Norte, Ceará, Brasil Fonte: Elaboração própria.

A UBSs rural Serra, de acordo com o Sistema de Informação da Atenção Básica, em 2014, possuía 22,45\% de sua população ingerindo água proveniente de poços, nascentes e outras fontes, assim como escassa coleta de esgoto doméstico (0,93\%).

Para Franco et al. (2012), a descarga de esgoto bruto in natura alcançará algum corpo hídrico, contaminando-o. Uma vez contaminada, a água opera como veículo de propagação de patógenos, pois possui grande capacidade de dispersão, por ser de uso abrangente e pelo fato de seu consumo ser obrigatório para a manutenção da vida.

Com relação a estudos que apliquem o SIG em territórios de UBSs, existem experiências em Montes Claros e Belo Horizonte, em Minas Gerais, Goiânia, Goiás (GO), Vitória da Conquista, Bahia (BA) e Caruaru, Pernambuco (PE) (Pereira; Barcellos, 2006). 
O uso do SIG possibilita indicar os riscos a que a população está exposta, acompanhar a disseminação dos agravos, fornecer subsídios para explicações causais, definir prioridades de intervenção e avaliar seu impacto, promovendo análises complexas, integração de dados de fontes distintas, além da criação de bancos de dados próprios com referências espaciais que podem ser aplicadas nas mais diversas áreas de estudos (Hino et al., 2006; Oliveira; Nascimento, 2009).

As razões que fazem do território Serra o mais vulnerável podem ser investigadas em estudos futuros, uma vez que vários podem ser os fatores intervenientes, como características do solo, perfil socioeconômico, qualidade da água e condições de saneamento, cujas variáveis podem ser analisadas mediante o uso do SIG.

\section{Considerações finais}

Observou-se que o enteroparasitismo em usuários do SUS, no município de Limoeiro do Norte, apresentou declínio ao longo de 2009 e 2014, sendo as mulheres e os adultos os mais acometidos, quando observadas as características pessoais dos indivíduos.

Quando verificado o ambiente onde reside o hospedeiro, constata-se que a infecção ocorre de maneira heterogênea, se concentrando na zona rural, onde o território adstrito à UBS Serra apresentou maior prevalência.

O uso do geoprocessamento pode ser de grande valia para futuros estudos que almejem realizar análises que, por exemplo, integrem indicadores socioeconômicos e ambientais em prol de um planejamento que vise o controle das parasitoses intestinais, uma vez que, a produção de mapas temáticos favorece a visualização de áreas de risco e, por conseguinte, onde estão as populações mais susceptíveis.

\section{Notas}

1 Cerca de $13,4 \%$ e $56,6 \%$ da população cearense tem déficit e/ou atendimento precário dos serviços de água e esgoto, respectivamente, cuja discrepância é visível comparando áreas urbanas (1,9\%) e rurais $(57,2 \%)$ dentro do Estado (IBGE, 2011). 


\section{Referências}

ABOSSIE, A; SEID, M. Assessment of the prevalence of intestinal parasitosis and associated risk factors among primary school children in Chencha town, Southern Ethiopia. BMC Public Health. v. 14, n. 166, p. 1-8, 2014.

ALVES, H. P. F. Desigualdade ambiental no município de São Paulo: análise da exposição diferenciada de grupos sociais a situações de risco ambiental através do uso de metodologias de geoprocessamento. Revista Brasileira de Estudos de População, v. 24, p. 301-316, 2007.

ALVES, J. R. et al. Parasitoses intestinais em região semi-árida do Nordeste do Brasil: resultados preliminares distintos das prevalências esperadas. Cadernos de Saúde Pública, v. 19, n. 2, p. 667-670, 2003.

ARCGIS 10. Software. Disponível em: <http://www.esri.com/software/arcgis>. Acesso em: 13 jul. 2014.

BASSO, R. M. C. et al. Evolução da prevalência de parasitoses intestinais em escolares em Caxias do Sul, RS. Revista da Sociedade Brasileira de Medicina Tropical, v. 41, n. 3, p. 263-268, 2008.

BELLIN, M.; GRAZZIOTIN, N. A. Prevalência de parasitos intestinais no município de Sanandauva/RS, Newslab, v. 18, n. 104, p. 116-122, 2011.

BELO, V.S. et al. Factors associated with intestinal parasitosis in a population of children and adolescents. Revista Paulista de Pediatria, v. 30, n. 2, p. 195-201, 2012.

BERMÚDEZ, A. et al. Enteroparasitismo, higiene y saneamiento ambiental en menores de seis comunidades indígenas. Cali-Colombia. Rev. salud pública. v. 15, n. 1, p. 1-11, 2013.

BORGES, W. F.; MARCIANO, F. M.; OLIVEIRA, H. B. Parasitos intestinais: elevada prevalência de Giardialamblia em pacientes atendidos pelo serviço público de saúde na região sudeste de Goiás, Brasil. Revista de Patologia Tropical, v. 40, n. 2, p. 149-157, 2011.

CASTRO, C. M. de; PEIXOTO, M. N. de O.; RIO, G. A. P. do. Riscos ambientais e Geografia: conceituações, abordagens e escalas. Anuário do Instituto de Geociências -UFRJ, v. 28. n. 2, p. 11-30. 2005.

CEARÁ. Governo do Estado do Ceará. Secretaria do Planejamento e Gestão. Perfil Básico Municipal, 2013.

CUNHA, G. M. et al. Prevalência de infecções por enteroparasitoses e sua relação com as condições socioeconômicas e ambientais em comunidades extrativistas no município de Cairu - Bahia. Revista Eletrônica de Engenharia Civil. v. 7, n. 2, p. 27-36, 2013.

FERNANDES, S. et al. Protocolo de parasitoses intestinais. Acta Pediátrica Portuguesa, v. 43, n. 1, p. 35-41, 2011.

FRANCO, R. M. B.; BRANCO, N.; LEAL, D. A. G. Parasitologia ambiental: métodos de concentração e detecção de Cryptosporidium spp. e Giardia spp. em amostras de água. Revista de Patologia Tropical (Online), v. 41, p. 119, 2012. 
FREGONESI, et al. Cryptosporidium e Giardia: desafios em água de abastecimento público. O Mundo da Saúde, v. 36, p. 602-609, 2012.

FREI, F.; JUNCANSEN, C.; RIBEIRO-PAES, J. T. Levantamento epidemiológico das parasitoses intestinais: viés analítico decorrente do tratamento profilático. Cad. Saúde Pública, v. 24, n. 12, p. 2919-2925, 2008.

GOMES, R; NASCIMENTO, E. F. ARAÚJO, F. C. Por que os homens buscam menos os serviços de saúde do que as mulheres? As explicações de homens com baixa escolaridade e homens com ensino superior. Caderno de Saúde Pública, v. 23, n. 3, p. 565-574, 2007.

HELLER, L. Saneamento e saúde pública. OPAS: Brasília, 1997.

HINO, P. et al. Distribuição espacial de doenças endêmicas no município de Ribeirão Preto, São Paulo. Ciência e Saúde Coletiva, ABRASCO, n. 128, v. 16, p. 1289-1294, 2011.

HINO, P. et al. Geoprocessing in health area. Revista Latino-Americana de Enfermagem, Ribeirão Preto, v. 14, p. 939-943, 2006.

HOFFMAN, W. A.; PONS, J. A.; JANER, J. L. Sedimentation concentration method in schistosomiasis mansoni. Puerto Rico J. Publ. Health \& Trop. Med, n. 9, p. 283-298, 1934.

IBGE. Atlas de saneamento 2011. Disponível em: <http:/www.ibge.gov.br/ home/estatistica/populacao/atlas_saneamento/default_zip.shtm >. Acesso em: 26 jul. 2013.

IBGE. Censo 2010. Disponível em: <http://www.censo2010.ibge.gov.br/>. Acesso em: 12 ago. 2010.

LIMOEIRO DO NORTE. Plano municipal de combate e erradicação da extrema pobreza, 2010

LIMOEIRO DO NORTE. Secretaria Municipal de Saúde. Info Hospi, Software. 2009

LODO, M. et al Prevalência de enteroparasitas em município do interior paulista. Revista Brasileira de Crescimento e Desenvolvimento Humano, v. 20, n. 3, p. 769777, 2010.

MAIA, C. V. A.; HASSUM, I. C.; VALLADARES, G. S. Fatores sociossanitários e parasitoses intestinais em Limoeiro do Norte - CE. Hygeia, v. 19, n. 10, p. 5064, 2014.

MASCARINI, L. M. et al. Impacto de um Programa de Saneamento Smbiental na prevalência e na incidência das parasitoses intestinais na população de idade escolar de Salvador. Revista VeraCidade, ano IV, n. 4, mar. 2009.

MATI, V. L. T.; PINTO, J. H. MELO, A. L. Levantamento de parasitoses intestinais nas áreas urbana e rural de Itambé do Mato Dentro, Minas Gerais, Brasil. Revista de Patologia Tropical, v. 40, n. 1, p. 92-100, 2011.

MATOS, M. A.; CRUZ, Z. V. Prevalência de parasitoses intestinais no município de Ibiassucê - Bahia. Revista Educação, Meio Ambiente e Saúde, v. 5, n. 1, p. 6471, 2012. 
MENEZES, R. A. D. et al. Enteroparasitoses em pacientes atendidos no laboratório de uma unidade de saúde de Macapá, Amapá, 2011 - 2012. Revista de Biologia e Ciências da Terra, v. 13, n. 1, p. 191-198, 2013.

NGUI, R. et al. Prevalence and risk factors of intestinal parasitism in rural and remote West Malaysia. PLoS Negl Trop Dis., v. 5, n. 3, e 974.p. 1-7, 2011.

OLIVEIRA, F. J. P.; NASCIMENTO, L. F. C. Análise espacial da distribuição de doenças diarréicas nos municípios do Vale do Paraíba Paulista. Ambi-Agua, Taubaté, v. 4, n. 2, p. 115-123, 2009.

OLIVEIRA, U. D.; CHIUCHETTA, S. J. R. Ocorrência de enteroparasitoses na população do município de Goioerê - PR. Estudos de Biologia, Ambiente e Diversidade, v. 31, n. 73/75, p. 81-85, 2009.

ORO, D. et al. Prevalência de parasitas intestinais em crianças de Descanso Santa Catarina - Brasil. Unoesc \& Ciência, v. 1, n. 2, p. 151-156, jul./dez. 2010.

PASCUAL, G. et al. Parásitos intestinales enpobladores de dos localidades de Yurimaguas, Alto Amazonas, Loreto, Perú. Neotrop. Helmintho, v. 4, n. 2, p.127136. jul./dez. 2010.

PEREIRA, M. P. B.; BARCELLOS, C. O território no Programa de Saúde da Família. Hygeia Revista Brasileira de Geografia Médica e da Saúde. v. 2, n. 2, p. 47-55, 2006.

PEZZI, N. C.; TAVARES, R. G. Relação de aspectos sócio-econômicos e ambientais com parasitoses intestinais e eosinofilia em crianças da ENCA, Caxias do SulRS. Revista Estudos, Goiânia, v. 34, n. 11-12, p. 1041-1055, nov./dez. 2007.

RBJA. Manifesto de lançamento da Rede Brasileira de Justiça Ambiental, 2002. Disponível em: <http://www.mma.gov.br/educacao-ambiental/politica-deeducacao-ambiental/documentos-referenciais/item/8077>. Acesso em: 10 jan. 2014.

ROCHA, T. J. M.; BRAZ, J. C.; CALHEIROS, C. M. L. Parasitismo intestinal em uma comunidade carente no município de Barra de Santo Antônio, Estado de Alagoas. Revista Eletrônica de Farmácia, v. 2, n. 3, p. 128-136, 2010.

SANTOS, A. S.; MERLINI, L. S. Prevalência de enteroparasitoses na população do município de Maria Helena, Paraná. Ciência Saúde Coletiva, v. 14, n. 3, 2010.

SANTOS, L. P.; SANTOS, F. L. N.; SOARES, N. M. Prevalência de parasitoses intestinais em pacientes atendidos no Hospital Universitário Professor Edgar Santos, Salvador - Bahia. Revista Brasileira de Patologia Tropical, v. 36, n. 3, p. 237- 246, 2007.

SILVA JUNIOR, J. R. Parasitoses intestinais e saneamento ambiental: estudo da situação dos bairros Bela Vista e Ulisses Guimarães em Teixeira de Freitas - BA. 2010. Dissertação (Mestrado Profissional em Tecnologias Ambientais) Faculdade de Aracruz, 2010.

SILVA, E. F. et al. Enteroparasitoses em crianças de áreas rurais do município de Coari, Amazonas, Brasil. Revista Brasileira de Patologia Tropical, v. 38, n. 1, p. 35-43, 2009. 
SILVA, F. S. et al. Frequência de parasitos intestinais no município de Chapadinha, Maranhão, Brasil. Revista Brasileira de Patologia Tropical, v. 39, n. 1, p. 63-68, 2010.

TEIXEIRA, J. C.; GOMES, M. H. R.; SOUZA, J. A. Estudo da associação entre cobertura por serviços de saneamento e indicadores epidemiológicos nos países da América Latina: estudo com dados secundários. Revista Panamericana de Salud Pública, v. 32, p. 419-425, 2012.

ZAIDEN, M. F. et al. Epidemiologia das parasitoses intestinais em crianças de creches de Rio Verde - GO. Revista da Faculdade de Medicina de Ribeirão Preto e do Hospital das Clínicas da FMRP, v. 41, n. 2, p. 182-187, abr./jun. 2008.

Carlos Vangerre de Almeida Maia - Tecnólogo em Recursos Hídricos/Saneamento Ambiental pelo Instituto Centro de Ensino Tecnológico do Ceará. Mestre em Desenvolvimento e Meio Ambiente pela Universidade Federal do Piauí. Atualmente, professor do Instituto Centro de Ensino Tecnológico do Ceará, lotado na Escola Estadual de Educação Profissional Francisca Rocha Silva, Jaguaruana, lecionando no Curso Técnico em Meio Ambiente.

Gustavo Souza Valladares - Engenheiro Agrônomo pela Universidade Federal Rural do Rio de Janeiro, Mestre e Doutor em Ciência do Solo pela mesma instituição. Atualmente é professor da Universidade Federal do Piauí. Trabalha com Ciência do Solo, Geografia Física e Geoprocessamento.

Izabella Cabral Hassum - Possui Graduação em Medicina-Veterinária pela Universidade Federal Rural do Rio de Janeiro. É Mestre em Parasitologia Veterinária e Doutora em Ciência Animal/Parasitologia pela mesma Universidade. Atualmente é pesquisadora A da Empresa Brasileira de Pesquisa Agropecuária, lotada no Centro de Pesquisa Agropecuária do Meio-Norte em Teresina/PI onde desenvolve trabalhos na área de Saúde Animal, subárea de Parasitologia.

Contribuição de cada autor no desenvolvimento do artigo:

Os autores ofereceram substanciais contribuições científicas e intelectuais ao estudo, participando, todos, do design do manuscrito. A coleta e análise dos dados, concepção, preparação e redação do manuscrito foram realizadas pelo primeiro autor. O segundo autor ficou especialmente responsável pelo desenvolvimento teórico-conceitual e metodológico, além da correção da redação A terceira autora ficou responsável pela revisão crítica do manuscrito e colaborou na interpretação dos dados epidemiológicos e na correção da redação.

Recebido para publicação em 18 de agosto de 2016 Aceito para publicação em 21 de setembro de 2016 